\title{
Analytical Solution for the Time-Dependent Emden-Fowler Type of Equations by Homotopy Analysis Method with Genetic Algorithm
}

\author{
Waleed Al-Hayani1, Laheeb Alzubaidy², Ahmed Entesar ${ }^{1}$ \\ ${ }^{1}$ Department of Mathematics, College of Computer Science and Mathematics, University of Mosul, Mosul, Iraq \\ ${ }^{2}$ Department of Software Engineering, College of Computer Science and Mathematics, University of Mosul, Mosul, Iraq \\ Email: waleedalhayani@yahoo.es
}

How to cite this paper: Al-Hayani, W., Alzubaidy, L. and Entesar, A. (2017) Analytical Solution for the Time-Dependent Emden-Fowler Type of Equations by Homotopy Analysis Method with Genetic Algorithm. Applied Mathematics, 8, 693-711. https://doi.org/10.4236/am.2017.85055

Received: December 30, 2016

Accepted: May 22, 2017

Published: May 25, 2017

Copyright ( 2017 by authors and Scientific Research Publishing Inc. This work is licensed under the Creative Commons Attribution International License (CC BY 4.0).

http://creativecommons.org/licenses/by/4.0/

\begin{abstract}
In this paper, Homotopy Analysis method with Genetic Algorithm is presented and used to obtain an analytical solution for the time-dependent Emden-Fowler type of equations and wave-type equation with singular behavior at $x=0$. The advantage of this single global method employed to present a reliable framework is utilized to overcome the singularity behavior at the point $x$ $=0$ for both models. The method is demonstrated for a variety of problems in one and higher dimensional spaces where approximate-exact solutions are obtained. The results obtained in all cases show the reliability and the efficiency of this method.
\end{abstract}

\section{Keywords}

Homotopy Analysis Method, Genetic Algorithm, Emden-Fowler Equation, Wave-Type Equation, Adomian Polynomials, Noise Terms, Padé Approximants, Simpson Rule

\section{Introduction}

In many previous works of the diffusion of heat perpendicular to the surfaces of parallel planes are modeled by the heat equation

$$
x^{-r}\left(x^{r} y_{x}\right)_{x}+a f(x, t) g(y)+h(x, t)=y_{t}, 0<x \leq L, 0<t<T, r>0,
$$

or equivalently

$$
y_{x x}+\frac{r}{x} y_{x}+a f(x, t) g(y)+h(x, t)=y_{t}, 0<x \leq L, 0<t<T, r>0,
$$

where $f(x, t) g(y)+h(x, t)$ is the nonlinear heat source, $y(x, t)$ is the tem- 
perature, and $t$ is the dimensionless time variable. For the steady-state case, and for $r=2, h(x, t)=0$, Equation (2) is the Emden-Fowler equation [1] [2] [3] given by

$$
y^{\prime \prime}+\frac{2}{x} y^{\prime}+a f(x) g(y)=0, \quad y(0)=y_{0}, y^{\prime}(0)=0,
$$

where $f(x)$ and $g(y)$ are some given functions of $x$ and $y$ respectively. For $f(x)=1$ and $g(y)=y^{n}$, Equation (3) is the standard Lane-Emden equation that was used to model the thermal behavior of a spherical cloud of gas acting under the mutual attraction of its molecules [1] and subject to the classical laws of thermodynamics. For other special forms of $g(y)$, the well-known LaneEmden equation was used to model several phenomena in mathematical physics and astrophysics such as the theory of stellar structure, the thermal behavior of a spherical cloud of gas, isothermal gas spheres, and theory of thermionic currents [1] [2] [3]. A substantial amount of work has been done on this type of problems for various structures of $g(y)$ in [1]-[7].

On the other hand, the wave type of equations with singular behavior is given by

$$
x^{-r}\left(x^{r} y_{x}\right)_{x}+a f(x, t) g(y)+h(x, t)=y_{t t}, 0<x \leq L, 0<t<T, r>0,
$$

or equivalently

$$
y_{x x}+\frac{r}{x} y_{x}+a f(x, t) g(y)+h(x, t)=y_{t t}, 0<x \leq L, 0<t<T, r>0,
$$

will be examined as well, where $f(x, t) g(y)+h(x, t)$ is a nonlinear source, $\mathrm{t}$ is the dimensionless time variable, and $y(x, t)$ is the displacement of the wave at the position $x$ and at time $t$. The singularity behavior that occurs at the point $x=0$ is the main difficulty in the analysis of Equations (2) and (5). Wazwaz [8] used the Adomian decomposition method (ADM) to get an analytical solution for the time-dependent Emden-Fowler type of equations and wave-type equation with singular behavior.

The main objective of this paper is to apply Homotopy Analysis Method (HAM) to obtain approximate-exact solutions for different models for the timedependent Emden-Fowler type of equations and wave-type equation with singular behavior at $x=0$. While the VIM [9] [10] requires the determination of Lagrange multiplier in its computational algorithm, HAM is independent of any such requirements, HAM handles linear and nonlinear terms in a simple and straightforward manner without any additional requirements. Also, in this paper we apply Genetic Algorithm (GA) to obtain an approximate solution of the same equations.

In what follows, we give a brief review of Homotopy analysis method and Genetic algorith.

\section{Analysis of the Homotopy Analysis Method}

To describe the basic ideas of the HAM, we consider the following differential 
equation:

$$
N[y(x, t)]=k(x, t),
$$

where $N$ is a nonlinear operator, $x$ and $t$ denote the independent variables, $y(x, t)$ is an unknown function and $k(x, t)$ is a known analytic function. For simplicity, we ignore all boundary or initial conditions, which can be treated in the similar way. By means of generalizing the traditional Homotopy method, Liao [11] constructs the so called zero-order deformation equation

$$
(1-q) L\left[\phi(x, t ; q)-y_{0}(x, t)\right]=q h[N[\phi(x, t ; q)]-k(x, t)],
$$

where $q \in[0,1]$ is an embedding parameter, $h$ is a nonzero auxiliary parameter, $L$ is an auxiliary linear operator, $y_{0}(x, t)$ is an initial guess of $y(x, t)$ and $\phi(x, t ; q)$ is an unknown function. It is important, that one has great freedom to choose auxiliary objects such as $h$ and $L$ in HAM. Obviously, when $q=0$ and $q=1$ it holds

$$
\phi(x, t ; 0)=y_{0}(x, t), \quad \phi(x, t ; 1)=y(x, t),
$$

respectively. Thus, as $q$ increases from 0 to 1 , the solution $\phi(x, t ; q)$ varies from the initial guess $y_{0}(x, t)$ to the solution $y(x, t)$. Expanding $\phi(x, t ; q)$ in Taylor series with respect to $q$, we have

$$
\phi(x, t ; q)=y_{0}(x, t)+\sum_{m=1}^{+\infty} y_{m}(x, t) q^{m},
$$

where

$$
y_{m}(x, t)=\left.\frac{1}{m !} \frac{\partial^{m} \phi(x, t ; q)}{\partial q^{m}}\right|_{q=0} .
$$

If the auxiliary linear operator, the initial guess, the auxiliary parameter $h$, and the auxiliary function are so properly chosen, the above series (9) converges at $q=1$, then we have

$$
\phi(x, t ; 1)=y_{0}(x, t)+\sum_{m=1}^{+\infty} y_{m}(x, t),
$$

which must be one of the solutions of the original nonlinear equation, as proved by Liao [11]. If $h=-1$, Equation (7) becomes

$$
(1-q) L\left[\phi(x, t ; q)-y_{0}(x, t)\right]+q[N[\phi(x, t ; q)]-k(x, t)]=0,
$$

which is used mostly in the Homotopy perturbation method [12].

According to Equation (10), the governing equation can be deduced from the zero-order deformation Equation (7). We define the vectors

$$
\boldsymbol{y}_{i}=\left\{y_{0}(x, t), y_{1}(x, t), \cdots, y_{i}(x, t)\right\} \text {. }
$$

Differentiating Equation (7) $m$ times with respect to the embedding parameter $q$ and then setting $q=0$ and finally dividing them by $m$ !, we have the socalled $m$ th-order deformation equation

$$
L\left[y_{m}(x, t)-\chi_{m} y_{m-1}(x, t)\right]=h R_{m}\left(\boldsymbol{y}_{m-1}\right),
$$


where

$$
R_{m}\left(\boldsymbol{x}_{m-1}\right)=\left.\frac{1}{(m-1) !} \frac{\partial^{m-1}\{N[\phi(x, t ; q)]-k(x, t)\}}{\partial q^{m-1}}\right|_{q=0},
$$

and

$$
\chi_{m}= \begin{cases}0, & m \leq 1 \\ 1, & m>1 .\end{cases}
$$

It should be emphasized that $y_{m}(x, t) \quad(m \geq 1)$ are governed by the linear Equation (14) with the linear boundary conditions that come from the original problem, which can be easily solved by symbolic computation softwares such as Maple and Mathematica.

\section{Genetic Algorithms}

Definition 1 Genetic Algorithms are search and optimization techniques based on Darwin's Principle of Natural Selection.

Definition 2 Genetic Algorithm Operators [13] [14]

The simplest form of genetic algorithm involves three types of operators: selection, crossover and mutation.

Selection: This operator selects chromosomes in the population for reproduction. The fitter the chromosome, the more times it is likely to be selected to reproduce.

Crossover: This operator randomly chooses a locus and exchanges the subsequences before and after that locus between two chromosomes to create two offspring. For example, the strings 10000100 and 1111111 could be crossed over after the third locus in each to produce the two offspring 10011111 and 11100100 .

The crossover operator roughly mimics biological recombination between two single-chromosome (haploid) organisms.

Mutation: This operator randomly flips some of the bits in a chromosome. For example, the string 00000100 might be mutated in its second position to yield 01000100 . Mutation can occur at each bit position in a string with some probability, usually very small (e.g., 0.001).

Algorithm 3 A Simple Genetic Algorithm [13] [14]

Given a clearly defined problem to be solved and a bit string representation for candidate solutions, a simple GA works as follows:

1 . Start with a randomly generated population of $n$ l-bit chromosomes (candidate solutions to a problem).

2. Calculate the fitness $f(x, t)$ of each chromosome $x$ and $t$ in the population.

3. Repeat the following steps until $n$ offspring have been created:

(a) Select a pair of parent chromosomes from the current population, the probability of selection being an increasing function of fitness. Selection is done "with replacement," meaning that the same chromosome can be selected more 
than once to become a parent.

(b) With probability $p c$ (the "crossover probability" or "crossover rate"), cross over the pair at randomly chosen point (chosen with uniform probability) to form two offspring. If no crossover takes place, form two offspring that are exact copies of their respective parents. (Note that here the crossover rate is defined to be the probability that two parents will crossover in a single point. There are also "multi-point crossover" versions of the GA in which the crossover rate for a pair of parents is the number of points at which a crossover takes place).

(c) Mutate the two offspring at each locus with probability pm (the "mutation probability" or "mutation rate"), and place the resulting chromosomes in the new population. If $\mathrm{n}$ is odd, one new population member can be discarded at random.

4. Replace the current population with the new population.

5. Go to step 2.

Also see the flow chart (Figure 1).

\section{Applications of the Method}

In this work, we examine six distinct models with singular behavior at $x=0$, two linear time-dependent Lane-Emden type of equations, two linear models of wave-type equation, and two nonlinear singular models. To show the high accuracy of the solution results compared with the exact solution, we give the numerical results applying the Genetic Algorithm (GA), HAM $(n=5)$, Padé approximants $(\mathrm{PA})$ of order $[p / q]$, and the numerical solution with the Simpson rule (SIMP). Twenty points have been used in the Simpson rule. The computations associated with the examples were performed using a Maple 13 package with a precision of 20 dgits.

\subsection{Time-Dependent Lane-Emden Type}

Example 1. Firstly, let us consider the following linear homogeneous equation

$$
y_{x x}+\frac{2}{x} y_{x}-\left(6+4 x^{2}-\cos t\right) y=y_{t},
$$

subject to initial conditions

$$
y(0, t)=\mathrm{e}^{\sin t}, \quad y_{x}(0, t)=0 .
$$

To solve Equations (17)-(18) by means of the standard HAM, we choose the initial approximation

$$
y_{0}(x, t)=\mathrm{e}^{\sin t}
$$

and the linear operator

$$
L[\phi(x, t ; q)]=\frac{\partial^{2} \phi(x, t ; q)}{\partial x^{2}}+\frac{2}{x} \frac{\partial \phi(x, t ; q)}{\partial x},
$$

with the property

$$
L\left[-\frac{c_{1}}{x}+c_{2}\right]=0
$$




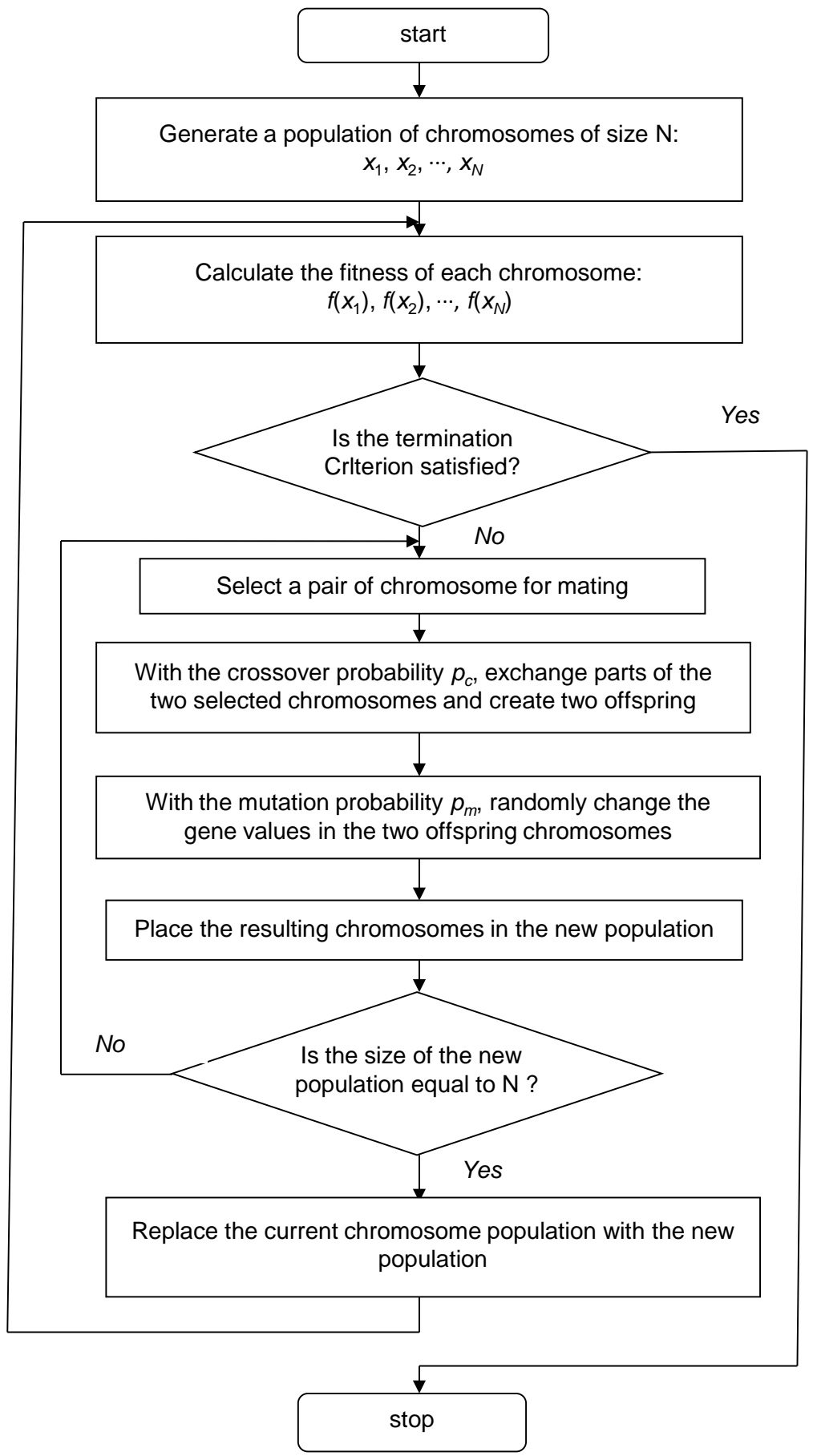

Figure 1. Flow Chart of Genetic Algorithm.

where $c_{i}(i=1,2)$ are constants of integration. Furthermore, Equation (17) suggests that we define the nonlinear operator as

$$
\begin{aligned}
N[\phi(x, t ; q)] & =\frac{\partial^{2} \phi(x, t ; q)}{\partial x^{2}}+\frac{2}{x} \frac{\partial \phi(x, t ; q)}{\partial x} \\
& -\left(6+4 x^{2}-\cos t\right) \phi(x, t ; q)-\frac{\partial \phi(x, t ; q)}{\partial t} .
\end{aligned}
$$

Using the above definition, we construct the zeroth-order deformation equa- 
tion as in (7) and (8) and the $m t h$-order deformation equation for $m \geq 1$ is

$$
L\left[y_{m}(x, t)-\chi_{m} y_{m-1}(x, t)\right]=h R_{m}\left(\boldsymbol{y}_{m-1}\right),
$$

with the initial conditions

$$
y_{m}(0, t)=0, \quad\left(y_{m}\right)_{x}(0, t)=0
$$

where

$$
R_{m}\left(\boldsymbol{y}_{m-1}\right)=\left(y_{m-1}\right)_{x x}+\frac{2}{x}\left(y_{m-1}\right)_{x}-\left(6+4 x^{2}-\cos t\right) y_{m-1}-\left(y_{m-1}\right)_{t} .
$$

Now, the solution of the $m t h$-order deformation Equation (23) for $m \geq 1$ is

$$
y_{m}(x, t)=\chi_{m} y_{m-1}(x, t)+h \int_{0}^{x} x^{-2} \int_{0}^{x} x^{2} R_{m}\left(y_{m-1}\right) .
$$

We now successively obtain

$$
\begin{aligned}
& y_{1}(x, t)=\left(-\frac{1}{5} h x^{4}-h x^{2}\right) \mathrm{e}^{\sin t}, \\
& y_{2}(x, t)=\left[\frac{1}{90} h^{2} x^{8}+\frac{13}{105} h^{2} x^{6}-\left(\frac{1}{5} h-\frac{1}{10} h^{2}\right) x^{4}-\left(h+h^{2}\right) x^{2}\right] \mathrm{e}^{\sin t}, \\
& y_{3}(x, t)=\left[-\frac{1}{3510} h^{3} x^{12}-\frac{59}{11550} h^{3} x^{10}+\left(\frac{1}{45} h^{2}-\frac{1}{210} h^{3}\right) x^{8}\right] \mathrm{e}^{\sin t} \\
& +\left[\left(\frac{26}{105} h^{2}+\frac{43}{210} h^{3}\right) x^{6}-\frac{1}{5}\left(h-h^{2}-2 h^{3}\right) x^{4}-\left(h+2 h^{2}+h^{3}\right) x^{2}\right] \mathrm{e}^{\sin t},
\end{aligned}
$$

and so on, in this manner the rest of the iterations can be obtained. Thus, the approximate solution in a series form is given by

$$
y(x, t)=y_{0}(x, t)+\sum_{m=1}^{+\infty} y_{m}(x, t),
$$

Hence, the series solution when $h=-1$ is

$$
y(x, t)=\left(1+x^{2}+\frac{1}{2 !} x^{4}+\frac{1}{3 !} x^{6}+\frac{1}{4 !} x^{8}+\cdots\right) \mathrm{e}^{\sin t} .
$$

This series has the closed form as $n \rightarrow \infty$

$$
y(x, t)=\mathrm{e}^{x^{2}+\sin t}
$$

which is the exact solution of (17)-(18) compatible with ADM [8].

Also, this example is solved by using GA as follows:

We'll choose six values of $x$ between $(0,1)$ randomly, and converting them from the decimal format to the binary format

$$
\begin{aligned}
& 0.2=\underbrace{0.001100}_{x_{1}} \underbrace{110011}_{t_{1}} \\
& 0.3=\underbrace{0.010011}_{x_{2}} \underbrace{001100}_{t_{2}} \\
& 0.5=\underbrace{0.100000}_{x_{3}} \underbrace{000000}_{t_{3}} \\
& 0.6=\underbrace{0.100110}_{x_{4}} \underbrace{011000}_{t_{4}}
\end{aligned}
$$




$$
\begin{aligned}
& 0.8=\underbrace{0.110011}_{x_{5}} \underbrace{001100}_{t_{5}} \\
& 0.9=\underbrace{0.111001}_{x_{6}} \underbrace{100100}_{t_{6}}
\end{aligned}
$$

that is,

\begin{tabular}{ccccccc}
\hline$x_{i}$ & 0.001100 & 0.010011 & 0.100000 & 0.100110 & 0.110011 & 0.111001 \\
$t_{i}$ & 0.110011 & 0.001100 & 0.000000 & 0.011000 & 0.011000 & 0.100100 \\
\hline
\end{tabular}

Now,

\begin{tabular}{cllllll}
\hline$x_{i}$ & 0.1875 & 0.2969 & 0.5000 & 0.5938 & 0.7969 & 0.8906 \\
$t_{i}$ & 0.7969 & 0.1875 & 0.0000 & 0.3750 & 0.1875 & 0.5625 \\
$f\left(x_{i}, t_{i}\right)$ & 2.1177 & 1.3159 & 1.2840 & 2.0521 & 2.2738 & 3.7675 \\
$p\left(x_{i}, t_{i}\right)$ & 0.1653 & 0.1027 & 0.1002 & 0.1602 & 0.1775 & 0.2941 \\
\hline
\end{tabular}

where $f\left(x_{i}, t_{i}\right)$ is the series solution of HAM given by (29) and $p\left(x_{i}, t_{i}\right)=$ $\frac{f\left(x_{i}, t_{i}\right)}{\sum_{i=1}^{6} f\left(x_{i}, t_{i}\right)}$ is the probability of each chromosomes with

$$
\sum_{i=1}^{6} f\left(x_{i}, t_{i}\right)=12.8110
$$

We arrange the chromosomes an ascending order and then choose less than four chromosomes to find the best solution

$$
\begin{array}{ll}
x_{31}=0.100000, & t_{31}=0.000000 \\
x_{21}=0.010011, & t_{21}=0.001100 \\
x_{41}=0.100110, & t_{41}=0.011000 \\
x_{11}=0.001100, & t_{11}=0.110011
\end{array}
$$

In this step of GA, crossover operation (two points) is done as follows:

$$
\begin{aligned}
& \text { cut }_{1}=2, \quad \text { cut }_{2}=5 \\
& x_{32}=0.100000, \quad t_{32}=0.001100 \\
& x_{22}=0.010011, \quad t_{22}=0.000000 \\
& x_{42}=0.101110, t_{42}=0.010000 \\
& x_{12}=0.000100, \quad t_{12}=0.111011
\end{aligned}
$$

In the last step of GA a mutation operation (bit inverse, $m=3$ ) and then converting them from the binary format to the decimal format:

$$
\begin{array}{ll}
x_{33}=0.101000=0.6250, & t_{33}=0.000100=0.0625 \\
x_{23}=0.011011=0.4219, & t_{23}=0.001000=0.1250 \\
x_{43}=0.100110=0.5938, & t_{43}=0.011000=0.3750 \\
x_{13}=0.001100=0.1875, & t_{13}=0.110011=0.7969
\end{array}
$$

The optimal solution is found after 51 generation to converge to the exact solution, where $x=7.2959306 E-04$ and $t=2.2287430 E-05$. After execute the Equation (29) many times by using GA as in Table 1 we found the optimal solution. 
Table 1. Optimal solution of genetic algorithm for example 1.

\begin{tabular}{ccccccc}
\hline$x$ & $t$ & $y_{\text {Exact }}(x, t)$ & $G A$ & $H A M(n=5)$ & $P A[6 / 6]$ & SIMP \\
\hline $7.296 \mathrm{E}-04$ & $2.229 \mathrm{E}-05$ & 1.0000228 & 1.0000228 & 1.0000228 & 1.0000228 & 1.0000228 \\
0.023 & 0.002 & 1.0025321 & 1.0023269 & 1.0025321 & 1.0025321 & 1.0025321 \\
0.029 & 0.031 & 1.0323482 & 1.0328346 & 1.0323482 & 1.0323482 & 1.0323482 \\
0.064 & 0.003 & 1.0071212 & 1.0074105 & 1.0071212 & 1.0071212 & 1.0071212 \\
0.222 & 0.011 & 1.0621379 & 1.0623118 & 1.0621379 & 1.0621379 & 1.0621379 \\
\hline
\end{tabular}

Example 2. We consider the following linear inhomogeneous equation,

$$
y_{x x}+\frac{2}{x} y_{x}-\left(5+4 x^{2}\right) y=y_{t}+6-5 x^{2}-4 x^{4},
$$

subject to initial conditions

$$
y(0, t)=\mathrm{e}^{t}, \quad y_{x}(0, t)=0 .
$$

To solve Equations (31)-(32) by means of the standard HAM, we choose the initial approximation

$$
y_{0}(x, t)=\mathrm{e}^{t},
$$

and the linear operator

$$
L[\phi(x, t ; q)]=\frac{\partial^{2} \phi(x, t ; q)}{\partial x^{2}}+\frac{2}{x} \frac{\partial \phi(x, t ; q)}{\partial x},
$$

with the property

$$
L\left[-\frac{c_{1}}{x}+c_{2}\right]=0
$$

where $c_{i} \quad(i=1,2)$ are constants of integration. Furthermore, Equation (31) suggests that we define the nonlinear operator as

$$
\begin{aligned}
N[\phi(x, t ; q)]= & \frac{\partial^{2} \phi(x, t ; q)}{\partial x^{2}}+\frac{2}{x} \frac{\partial \phi(x, t ; q)}{\partial x}-\left(5+4 x^{2}\right) \phi(x, t ; q) \\
& -\frac{\partial \phi(x, t ; q)}{\partial t}-\left(6-5 x^{2}-4 x^{4}\right) .
\end{aligned}
$$

Using the above definition, we construct the zeroth-order deformation equation as in (7) and (8) and the $m$ th-order deformation equation for $m \geq 1$ is

$$
L\left[y_{m}(x, t)-\chi_{m} y_{m-1}(x, t)\right]=h R_{m}\left(y_{m-1}\right),
$$

with the initial conditions

$$
y_{m}(0, t)=0, \quad\left(y_{m}\right)_{x}(0, t)=0
$$

where

$$
\begin{aligned}
R_{m}\left(y_{m-1}\right)= & \left(y_{m-1}\right)_{x x}+\frac{2}{x}\left(y_{m-1}\right)_{x}-\left(5+4 x^{2}\right) y_{m-1} \\
& -\left(y_{m-1}\right)_{t}-\left(1-\chi_{m}\right)\left(6-5 x^{2}-4 x^{4}\right) .
\end{aligned}
$$

Now, the solution of the $m$ th-order deformation Equation (37) for $m \geq 1$ is 


$$
y_{m}(x, t)=\chi_{m} y_{m-1}(x, t)+h \int_{0}^{x} x^{-2} \int_{0}^{x} x^{2} R_{m}\left(y_{m-1}\right) .
$$

We now successively obtain

$$
\begin{aligned}
y_{1}(x, t)= & -\left(\frac{1}{5} x^{4}+x^{2}\right) h \mathrm{e}^{t}+\left(\frac{2}{21} x^{6}+\frac{1}{4} x^{4}-x^{2}\right) h, \\
y_{2}(x, t)= & \left(\frac{1}{90} h^{2} x^{8}+\frac{13}{105} h^{2} x^{6}+\frac{1}{10} h^{2} x^{4}-\frac{1}{5} h x^{4}-h^{2} x^{2}-h x^{2}\right) \mathrm{e}^{t}-\frac{4}{1155} h^{2} x^{10} \\
& -\frac{31}{1512} h^{2} x^{8}+\left(\frac{9}{56} h^{2}+\frac{2}{21} h\right) x^{6}+\left(\frac{1}{2} h^{2}+\frac{1}{4} h\right) x^{4}-\left(h^{2}+h\right) x^{2},
\end{aligned}
$$

and so on, in this manner the rest of the iterations can be obtained. Thus, the approximate solution in a series form is given by

$$
y(x, t)=y_{0}(x, t)+\sum_{m=1}^{+\infty} y_{m}(x, t),
$$

Hence, the series solution when $h=-1$ is given by

$$
y(x, t)=x^{2}+\left(1+x^{2}+\frac{1}{2 !} x^{4}+\frac{1}{3 !} x^{6}+\frac{1}{4 !} x^{8}+\cdots\right) \mathrm{e}^{t}+\text { noise terms. }
$$

This series has the closed form as $n \rightarrow \infty$

$$
y(x, t)=x^{2}+\mathrm{e}^{x^{2}+t},
$$

which is the exact solution of the problem (31)-(32) compatible with ADM [8]. Notice that the noise terms that appear between various components vanish in the limit.

Using GA by the same procedure as in example 1, we get the optimal solution is found after 51 generation to converge to the exact solution, where $x=$ $4.9489 E-05$ and $t=7.6780 E-05$. After execute the Equation (43) many times by using GA as in Table 2 we found the optimal solution.

\subsection{Singular Wave-Type Equations}

Example 3. Now, we consider the inhomogeneous singular wave-type equation,

$$
y_{x x}+\frac{2}{x} y_{x}-\left(5+4 x^{2}\right) y=y_{t t}+12 x-5 x^{3}-4 x^{5},
$$

subject to initial conditions

Table 2. Optimal solution of genetic algorithm for example 2.

\begin{tabular}{ccccccc}
\hline$x$ & $t$ & $y_{\text {Exact }}(x, t)$ & $G A$ & $H A M(n=5)$ & $P A[6 / 6]$ & SIMP \\
\hline $4.949 \mathrm{E}-05$ & $7.678 \mathrm{E}-05$ & 1.0000767 & 1.0000767 & 1.0000767 & 1.0000767 & 1.0000767 \\
0.000 & 0.085 & 1.0887170 & 1.0882976 & 1.0887170 & 1.0887170 & 1.0887170 \\
0.065 & 0.023 & 1.0318239 & 1.0322448 & 1.0323482 & 1.0323482 & 1.0323482 \\
0.125 & 0.002 & 1.0334062 & 1.0333424 & 1.0334062 & 1.0334062 & 1.0334062 \\
0.185 & 0.175 & 1.2669473 & 1.2667575 & 1.2669473 & 1.2669473 & 1.2669473 \\
\hline
\end{tabular}




$$
y(0, t)=\mathrm{e}^{-t}, \quad y_{x}(0, t)=0 .
$$

To solve Equations (45)-(46) by means of the standard HAM, we choose the initial approximation

$$
y_{0}(x, t)=\mathrm{e}^{-t},
$$

and the linear operator

$$
L[\phi(x, t ; q)]=\frac{\partial^{2} \phi(x, t ; q)}{\partial x^{2}}+\frac{2}{x} \frac{\partial \phi(x, t ; q)}{\partial x},
$$

with the property

$$
L\left[-\frac{c_{1}}{x}+c_{2}\right]=0,
$$

where $c_{i}(i=1,2)$ are constants of integration. Furthermore, Equation (45) suggests that we define the nonlinear operator as

$$
\begin{aligned}
N[\phi(x, t ; q)]= & \frac{\partial^{2} \phi(x, t ; q)}{\partial x^{2}}+\frac{2}{x} \frac{\partial \phi(x, t ; q)}{\partial x}-\left(5+4 x^{2}\right) \phi(x, t ; q) \\
& -\frac{\partial^{2} \phi(x, t ; q)}{\partial t^{2}}-\left(12 x-5 x^{3}-4 x\right) .
\end{aligned}
$$

Using the above definition, we construct the zeroth-order deformation equation as in (7) and (8) and the $m$ th-order deformation equation for $m \geq 1$ is

$$
L\left[y_{m}(x, t)-\chi_{m} y_{m-1}(x, t)\right]=h R_{m}\left(y_{m-1}\right),
$$

with the initial conditions

$$
y_{m}(0, t)=0, \quad\left(y_{m}\right)_{x}(0, t)=0
$$

where

$$
\begin{aligned}
R_{m}\left(\boldsymbol{y}_{m-1}\right) & =\left(y_{m-1}\right)_{x x}+\frac{2}{x}\left(y_{m-1}\right)_{x}-\left(5+4 x^{2}\right) y_{m-1} \\
& -\left(y_{m-1}\right)_{t t}-\left(1-\chi_{m}\right)\left(12 x-5 x^{3}-4 x^{4}\right) .
\end{aligned}
$$

Now, the solution of the $m t h$-order deformation Equation (50) for $m \geq 1$ is

$$
y_{m}(x, t)=\chi_{m} y_{m-1}(x, t)+h \int_{0}^{x} x^{-2} \int_{0}^{x} x^{2} R_{m}\left(y_{m-1}\right) .
$$

We now successively obtain

$$
\begin{aligned}
y_{1}(x, t)= & -\left(\frac{1}{5} x^{4}+x^{2}\right) h \mathrm{e}^{-t}+\left(\frac{1}{14} x^{7}+\frac{1}{6} x^{5}-x^{3}\right) h, \\
y_{2}(x, t)= & \left(\frac{1}{90} h^{2} x^{8}+\frac{13}{105} h^{2} x^{6}+\frac{1}{10} h^{2} x^{4}-\frac{1}{5} h x^{4}-h^{2} x^{2}-h x^{2}\right) \mathrm{e}^{-t}-\frac{1}{462} h^{2} x^{11} \\
& -\frac{43}{3780} h^{2} x^{9}+\left(\frac{43}{336} h^{2}+\frac{1}{14} h\right) x^{7}+\left(\frac{1}{3} h^{2}+\frac{1}{6} h\right) x^{5}-\left(h^{2}+h\right) x^{3},
\end{aligned}
$$

and so on, in this manner the rest of the iterations can be obtained. Thus, the approximate solution in a series form is given by 


$$
y(x, t)=y_{0}(x, t)+\sum_{m=1}^{+\infty} y_{m}(x, t),
$$

Hence, the series solution when $h=-1$ is given by

$$
y(x, t)=x^{3}+\left(1+x^{2}+\frac{1}{2 !} x^{4}+\frac{1}{3 !} x^{6}+\frac{1}{4 !} x^{8}+\cdots\right) \mathrm{e}^{-t}+\text { noise terms. }
$$

This series has the closed form as $n \rightarrow \infty$

$$
y(x, t)=x^{3}+\mathrm{e}^{x^{2}-t},
$$

which is the exact solution of the problem (45)-(46) compatible with ADM [8]. Notice that the noise terms that appear between various components vanish in the limit.

Using GA by the same procedure as in example 1, we get the optimal solution is found after 51 generation to converge to the exact solution, where $x=0$ and $t=0.879$. After execute the Equation (56) many times by using GA as in Table 3 we found the optimal solution

Example 4. We now examine the inhomogeneous wave-type equation,

$$
y_{x x}+\frac{4}{x} y_{x}-\left(18 x+9 x^{4}\right) y=y_{t t}-2-\left(18 x+9 x^{4}\right) t^{2},
$$

subject to initial conditions

$$
y(0, t)=1+t^{2}, \quad y_{x}(0, t)=0 .
$$

To solve Equations (58)-(59) by means of the standard HAM, we choose the initial approximation

$$
y_{0}(x, t)=1+t^{2}
$$

and the linear operator

$$
L[\phi(x, t ; q)]=\frac{\partial^{2} \phi(x, t ; q)}{\partial x^{2}}+\frac{4}{x} \frac{\partial \phi(x, t ; q)}{\partial x},
$$

with the property

$$
L\left[-\frac{c_{1}}{3 x}+c_{2}\right]=0
$$

where $c_{i} \quad(i=1,2)$ are constants of integration. Furthermore, Equation (58) suggests that we define the nonlinear operator as

Table 3. Optimal solution of genetic algorithm for example 3.

\begin{tabular}{ccccccc}
\hline$x$ & $t$ & $Y_{\text {Exact }}(x, t)$ & $G A$ & $H A M(n=5)$ & $P A[5 / 5]$ & SIMP \\
\hline 0.000 & 0.879 & 0.4151979 & 0.4150192 & 0.4151979 & 0.4151979 & 0.4151979 \\
0.011 & 0.872 & 0.4181664 & 0.4183309 & 0.4181664 & 0.4181664 & 0.4181664 \\
0.092 & 0.845 & 0.4339872 & 0.4338056 & 0.4339872 & 0.4339872 & 0.4339872 \\
0.136 & 0.901 & 0.4400918 & 0.4161477 & 0.4400918 & 0.4400918 & 0.4400918 \\
0.203 & 0.961 & 0.4069677 & 0.4071075 & 0.4069677 & 0.4069677 & 0.4069677 \\
\hline
\end{tabular}




$$
\begin{aligned}
N[\phi(x, t ; q)]= & \frac{\partial^{2} \phi(x, t ; q)}{\partial x^{2}}+\frac{4}{x} \frac{\partial \phi(x, t ; q)}{\partial x}-\left(18 x+9 x^{4}\right) \phi(x, t ; q) \\
& -\frac{\partial^{2} \phi(x, t ; q)}{\partial t^{2}}+\left(18 x+9 x^{4}\right) t^{2}+2 .
\end{aligned}
$$

Using the above definition, we construct the zeroth-order deformation equation as in (7) and (8) and the $m$ th-order deformation equation for $m \geq 1$ is

$$
L\left[y_{m}(x, t)-\chi_{m} y_{m-1}(x, t)\right]=h R_{m}\left(y_{m-1}\right),
$$

with the initial conditions

$$
y_{m}(0, t)=0, \quad\left(y_{m}\right)_{x}(0, t)=0
$$

where

$$
\begin{aligned}
R_{m}\left(\boldsymbol{y}_{m-1}\right) & =\left(y_{m-1}\right)_{x x}+\frac{4}{x}\left(y_{m-1}\right)_{x}-\left(18 x+9 x^{4}\right) y_{m-1} \\
& -\left(y_{m-1}\right)_{t t}+\left(1-\chi_{m}\right)\left[\left(18 x+9 x^{4}\right) t^{2}+2\right] .
\end{aligned}
$$

Now, the solution of the $m t h$-order deformation Equation (64) for $m \geq 1$ is

$$
y_{m}(x, t)=\chi_{m} y_{m-1}(x, t)+h \int_{0}^{x} x^{-4} \int_{0}^{x} x^{4} R_{m}\left(y_{m-1}\right) .
$$

We now successively obtain

$$
\begin{aligned}
y_{1}(x, t)= & -\frac{1}{6} h x^{6}-h x^{3} \\
y_{2}(x, t)= & \frac{1}{120} h^{2} x^{12}+\frac{1}{9} h^{2} x^{9}+\frac{1}{6}\left(h^{2}-h\right) x^{6}-\left(h^{2}+h\right) x^{3} \\
y_{3}(x, t)= & -\frac{1}{5040} h^{3} x^{18}-\frac{23}{5400} h^{3} x^{15}-\left(\frac{1}{90} h^{3}-\frac{1}{60} h^{2}\right) x^{12}+\left(\frac{1}{6} h^{3}+\frac{2}{9} h^{2}\right) x^{9} \\
& +\frac{1}{6}\left(3 h^{3}+2 h^{2}-h\right) x^{6}-\left(h^{3}+2 h^{2}+h\right) x^{3}
\end{aligned}
$$

and so on, in this manner the rest of the iterations can be obtained. Thus, the approximate solution in a series form is given by

$$
y(x, t)=y_{0}(x, t)+\sum_{m=1}^{+\infty} y_{m}(x, t),
$$

Thus, the series solution when $h=-1$ is given by

$$
y(x, t)=t^{2}+\left(1+x^{3}+\frac{1}{2 !} x^{6}+\frac{1}{3 !} x^{9}+\frac{1}{4 !} x^{12}+\cdots\right) .
$$

This series has the closed form as $n \rightarrow \infty$

$$
y(x, t)=t^{2}+\mathrm{e}^{x^{3}},
$$

which is the exact solution of the problem (58)-(59) compatible with ADM [8].

Using GA by the same procedure as in example 1, we get the optimal solution is found after 51 generation to converge to the exact solution, where $x=$ $4.6903 E-06$ and $t=1.1498 E-06$. After execute the Equation (70) many times by using GA as in Table 4 we found the optimal solution 
Table 4. Optimal solution of genetic algorithm for example 4.

\begin{tabular}{ccccccc}
\hline$x$ & $t$ & $y_{\text {Exact }}(x, t)$ & $G A$ & $H A M(n=5)$ & $P A[6 / 6]$ & SIMP \\
\hline $4.693 \mathrm{E}-06$ & $1.150 \mathrm{E}-06$ & 1.0000000 & 1.0000000 & 1.0000000 & 1.0000000 & 1.0000000 \\
0.000 & 0.102 & 1.0104040 & 1.0103960 & 1.0104040 & 1.0104040 & 1.0104040 \\
0.058 & 0.035 & 1.0014201 & 1.0013941 & 1.0014201 & 1.0014201 & 1.0014201 \\
0.164 & 0.079 & 1.0106616 & 1.0106261 & 1.0106616 & 1.0106616 & 1.0106616 \\
0.173 & 0.001 & 1.0051921 & 1.0051844 & 1.0051921 & 1.0051921 & 1.0051921 \\
\hline
\end{tabular}

\subsection{Nonlinear Models}

In what follows we close this analysis by examining two nonlinear time-dependent equations

Example 5. We now consider the nonlinear time-dependent equation,

$$
y_{x x}+\frac{5}{x} y_{x}+\left(24 t+16 t^{2} x^{2}\right) \mathrm{e}^{y}-2 x^{2} \mathrm{e}^{\frac{y}{2}}=y_{t},
$$

subject to initial conditions

$$
y(0, t)=0, \quad y_{x}(0, t)=0 .
$$

To solve Equations (72)-(73) by means of the standard HAM, we choose the initial approximation

$$
y_{0}(x, t)=0,
$$

and the linear operator

$$
L[\phi(x, t ; q)]=\frac{\partial^{2} \phi(x, t ; q)}{\partial x^{2}}+\frac{5}{x} \frac{\partial \phi(x, t ; q)}{\partial x},
$$

with the property

$$
L\left[-\frac{c_{1}}{4 x}+c_{2}\right]=0
$$

where $c_{i}(i=1,2)$ are constants of integration. Furthermore, Equation (72) suggests that we define the nonlinear operator as

$$
\begin{aligned}
N[\phi(x, t ; q)]= & \frac{\partial^{2} \phi(x, t ; q)}{\partial x^{2}}+\frac{5}{x} \frac{\partial \phi(x, t ; q)}{\partial x}+\left(24 t+16 t^{2} x^{2}\right) \mathrm{e}^{\phi(x, t ; q)} \\
& -2 x^{2} \mathrm{e}^{\frac{\phi(x, t ; q)}{2}}-\frac{\partial \phi(x, t ; q)}{\partial t} .
\end{aligned}
$$

Using the above definition, we construct the zeroth-order deformation equation as in (7) and (8) and the $m$ th-order deformation equation for $m \geq 1$ is

$$
L\left[y_{m}(x, t)-\chi_{m} y_{m-1}(x, t)\right]=h R_{m}\left(\boldsymbol{y}_{m-1}\right),
$$

with the initial conditions

$$
y_{m}(0, t)=0, \quad\left(y_{m}\right)_{x}(0, t)=0
$$

where 


$$
R_{m}\left(\boldsymbol{y}_{m-1}\right)=\left(y_{m-1}\right)_{x x}+\frac{5}{x}\left(y_{m-1}\right)_{x}+\left(24 t+16 t^{2} x^{2}\right) A_{m-1}-2 x^{2} B_{m-1}-\left(y_{m-1}\right)_{t} .
$$

For the nonlinear terms $\mathrm{e}^{\phi(x, t ; q)}=\sum_{m=1}^{\infty} A_{m-1}$ and $\mathrm{e}^{\frac{\phi(x, t ; q)}{2}}=\sum_{m=1}^{\infty} B_{m-1}$, the corresponding Adomian polynomials [15] [16] are:

$$
\begin{aligned}
A_{0} & =\mathrm{e}^{y_{0}}, \\
A_{1} & =y_{1} \mathrm{e}^{y_{0}}, \\
A_{2} & =\left[y_{2}+\left(\frac{1}{2 !}\right) y_{1}^{2}\right] \mathrm{e}^{y_{0}}, \\
A_{3} & =\left[y_{3}+y_{1} y_{2}+\left(\frac{1}{3 !}\right) y_{1}^{3}\right] \mathrm{e}^{y_{0}}, \\
A_{4} & =\left[y_{4}+\left(\frac{1}{2 !}\right) y_{2}^{2}+y_{1} y_{3}+\left(\frac{1}{2 !}\right) y_{1}^{2} y_{2}+\left(\frac{1}{4 !}\right) y_{1}^{4}\right] \mathrm{e}^{y_{0}}, \\
& \vdots
\end{aligned}
$$

and

$$
\begin{aligned}
& B_{0}=\mathrm{e}^{y_{0} / 2}, \\
& B_{1}=\frac{1}{2} y_{1} \mathrm{e}^{y_{0} / 2}, \\
& B_{2}=\left[\frac{1}{2} y_{2}+\frac{1}{4}\left(\frac{1}{2 !}\right) y_{1}^{2}\right] \mathrm{e}^{y_{0} / 2}, \\
& B_{3}=\left[\frac{1}{2} y_{3}+\frac{1}{4} y_{1} y_{2}+\frac{1}{8}\left(\frac{1}{3 !}\right) y_{1}^{3}\right] \mathrm{e}^{y_{0} / 2}, \\
& B_{4}=\left[\frac{1}{2} y_{4}+\frac{1}{4}\left(\frac{1}{2 !} y_{2}^{2}+\frac{1}{4} y_{1} y_{3}\right)+\frac{1}{8}\left(\frac{1}{2 !}\right) y_{1}^{2} y_{2}+\frac{1}{16}\left(\frac{1}{4 !}\right) y_{1}^{4}\right] \mathrm{e}^{y_{0} / 2},
\end{aligned}
$$

Now, the solution of the $m t h$-order deformation Equation (78) for $m \geq 1$ is

$$
y_{m}(x, t)=\chi_{m} y_{m-1}(x, t)+h \int_{0}^{x} x^{-5} \int_{0}^{x} x^{5} R_{m}\left(y_{m-1}\right) .
$$

We now successively obtain

$$
\begin{aligned}
y_{1}(x, t)= & \frac{1}{2} h t^{2} x^{4}-\frac{1}{16} h x^{4}+2 h t x^{2} \\
y_{2}(x, t)= & \left(\frac{1}{12} h^{2} t^{4}-\frac{1}{64} h^{2} t^{2}+\frac{1}{1536} h^{2}\right) x^{8}+\left(\frac{11}{15} h^{2} t^{3}-\frac{3}{40} h^{2} t\right) x^{6} \\
& +\left(2 h^{2} t^{2}+\frac{1}{2} h t^{2}-\frac{1}{8} h^{2}-\frac{1}{16} h\right) x^{4}+2\left(h^{2}+h\right) t x^{2}
\end{aligned}
$$

and so on, in this manner the rest of the iterations can be obtained. Thus, the approximate solution in a series form is given by

$$
y(x, t)=y_{0}(x, t)+\sum_{m=1}^{+\infty} y_{m}(x, t)
$$

Thus, the series solution when $h=-1$ is given by 


$$
y(x, t)=-2\left(t x^{2}-\frac{1}{2} t^{2} x^{4}+\frac{1}{3} t^{3} x^{6}-\frac{1}{4} t^{4} x^{8}+\cdots\right)+\text { noise terms. }
$$

This series has the closed form as $n \rightarrow \infty$

$$
y(x, t)=-2 \ln \left(1+t x^{2}\right),
$$

which is the exact solution of the problem (72)-(73) compatible with ADM [8]. Notice that the noise terms that appear between various components vanish in the limit.

Using GA by the same procedure as in example 1, we get the optimal solution is found after 51 generation to converge to the exact solution, where $x=0.470$ and $t=0.895$. After execute the Equation (85) many times by using GA as in Table 5 we found the optimal solution

Example 6. Finally, we examine the nonlinear homogeneous equation,

$$
y_{x x}+\frac{6}{x} y_{x}+\left(14 t+x^{4}\right) y+4 t y \ln y=y_{t t},
$$

subject to initial conditions

$$
y(0, t)=1, \quad y_{x}(0, t)=0 .
$$

To solve Equations (87)-(88) by means of the standard HAM, we choose the initial approximation

$$
y_{0}(x, t)=1 \text {, }
$$

and the linear operator

$$
L[\phi(x, t ; q)]=\frac{\partial^{2} \phi(x, t ; q)}{\partial x^{2}}+\frac{6}{x} \frac{\partial \phi(x, t ; q)}{\partial x},
$$

with the property

$$
L\left[-\frac{c_{1}}{5 x}+c_{2}\right]=0
$$

where $c_{i}(i=1,2)$ are constants of integration. Furthermore, Equation (87) suggests that we define the nonlinear operator as

$$
\begin{aligned}
N[\phi(x, t ; q)]= & \frac{\partial^{2} \phi(x, t ; q)}{\partial x^{2}}+\frac{6}{x} \frac{\partial \phi(x, t ; q)}{\partial x}+\left(14 t+x^{4}\right) \phi(x, t ; q) \\
& +4 t \phi(x, t ; q) \ln \phi(x, t ; q)-\frac{\partial^{2} \phi(x, t ; q)}{\partial t^{2}} .
\end{aligned}
$$

Table 5. Optimal solution of genetic algorithm for example 5.

\begin{tabular}{cccccc}
\hline$x$ & $t$ & $Y_{\text {Exact }}(x, t)$ & $G A$ & $H A M(n=5)$ & $P A[6 / 6]$ \\
\hline 0.470 & 0.895 & -0.3608152 & -0.3606302 & -0.3603871 & -0.3608131 \\
0.841 & 0.813 & -0.9085352 & -0.8831179 & -0.8170968 & -0.9083255 \\
0.866 & 0.878 & -1.0117805 & -0.9155520 & -0.8245006 & -1.0114234 \\
0.946 & 0.728 & -1.0033665 & -0.9260635 & -0.8376409 & -1.0030238 \\
0.974 & 0.672 & -0.9863539 & -0.9277153 & -0.8436189 & -0.9860390 \\
\hline
\end{tabular}


Using the above definition, we construct the zeroth-order deformation equation as in (7) and (8) and the $m$ th-order deformation equation for $m \geq 1$ is

$$
L\left[y_{m}(x, t)-\chi_{m} y_{m-1}(x, t)\right]=h R_{m}\left(y_{m-1}\right),
$$

with the initial conditions

$$
y_{m}(0, t)=0, \quad\left(y_{m}\right)_{x}(0, t)=0
$$

where

$$
R_{m}\left(\boldsymbol{y}_{m-1}\right)=\left(y_{m-1}\right)_{x x}+\frac{6}{x}\left(y_{m-1}\right)_{x}+\left(14 t+x^{4}\right) y_{m-1}+4 t A_{m-1}-\left(y_{m-1}\right)_{t t} .
$$

For the nonlinear term $\phi(x, t ; q) \ln \phi(x, t ; q)=\sum_{m=1}^{\infty} A_{m-1}$, the corresponding Adomian polynomials [15] [16] are:

$$
\begin{aligned}
& A_{0}=y_{0} \ln y_{0}, \\
& A_{1}=\left(1+\ln y_{0}\right) y_{1}, \\
& A_{2}=\left(1+\ln y_{0}\right) y_{2}+\frac{1}{y_{0}}\left(\frac{1}{2 !}\right) y_{1}^{2}, \\
& A_{3}=\left(1+\ln y_{0}\right) y_{3}+\frac{1}{y_{0}} y_{1} y_{2}-\frac{1}{y_{0}^{2}}\left(\frac{1}{3 !}\right) y_{1}^{3}, \\
& A_{4}=\left(1+\ln y_{0}\right) y_{4}+\frac{1}{y_{0}}\left(\frac{1}{2 !} y_{2}^{2}+y_{1} y_{3}\right)-\frac{1}{y_{0}^{2}}\left(\frac{1}{2 !}\right) y_{1}^{2} y_{2}+\frac{2}{y_{0}^{3}}\left(\frac{1}{4 !}\right) y_{1}^{4},
\end{aligned}
$$

Now, the solution of the $m t h$-order deformation Equation (93) for $m \geq 1$ is

$$
y_{m}(x, t)=\chi_{m} y_{m-1}(x, t)+h \int_{0}^{x} x^{-6} \int_{0}^{x} x^{6} R_{m}\left(y_{m-1}\right) .
$$

We now successively obtain

$$
\begin{aligned}
y_{1}(x, t)= & \frac{1}{66} h x^{6}+h t x^{2}, \\
y_{2}(x, t)= & \frac{1}{13464} h^{2} x^{12}+\frac{7}{572} h^{2} t x^{8}+\frac{1}{66}\left(h^{2}+h\right) x^{6}+\frac{1}{2} h^{2} t^{2} x^{4}+\left(h^{2}+h\right) t x^{2}, \\
y_{3}(x, t)= & \frac{1}{5574096} h^{3} x^{18}+\frac{965}{18290844} h^{3} t x^{14}+\frac{1}{6732}\left(h^{3}+h^{2}\right) x^{12} \\
& +\frac{67}{12870} h^{3} t^{2} x^{10}+\frac{7}{286}\left(h^{3}+h^{2}\right) t x^{8}+\frac{1}{66}\left(11 h^{3} t^{3}+2 h^{2}+h\right) x^{6} \\
& +\left(h^{3}+h^{2}\right) t^{2} x^{4}+\left(h^{3}+2 h^{2}+h\right) t x^{2}
\end{aligned}
$$

and so on, in this manner the rest of the iterations can be obtained. Thus, the approximate solution in a series form is given by

$$
y(x, t)=y_{0}(x, t)+\sum_{m=1}^{+\infty} y_{m}(x, t),
$$

Thus, the series solution when $h=-1$ is given by

$$
y(x, t)=1-t x^{2}+\frac{1}{2 !} t^{2} x^{4}-\frac{1}{3 !} t^{3} x^{6}+\frac{1}{4 !} t^{4} x^{8}+\cdots .
$$


Table 6. Optimal solution of genetic algorithm for example 6.

\begin{tabular}{cccccc}
\hline$x$ & $t$ & $y_{\text {Exact }}(x, t)$ & $G A$ & $H A M(n=5)$ & $P A[6 / 6]$ \\
\hline 0.270 & 0.935 & 0.8117150 & 0.8116540 & 0.8117157 & 0.8117155 \\
0.478 & 0.913 & 1.0025321 & 1.0023269 & 1.0025321 & 1.0025321 \\
0.855 & 0.954 & 0.4978791 & 0.4974576 & 0.4983086 & 0.4979100 \\
0.903 & 0.973 & 0.4523075 & 0.4521125 & 0.4532268 & 0.4522629 \\
0.981 & 0.938 & 0.4054755 & 0.4052946 & 0.4069536 & 0.4057609 \\
\hline
\end{tabular}

This series has the closed form as $n \rightarrow \infty$

$$
y(x, t)=\mathrm{e}^{-t x^{2}},
$$

which is the exact solution of the problem (87)-(88) compatible with ADM [8].

Using GA by the same procedure as in example 1, we get the optimal solution is found after 51 generation to converge to the exact solution, where $x=0.270$ and $t=0.935$. After execute the Equation (100) many times by using GA as in Table 6 we found the optimal solution.

\section{Conclusions}

To our best knowledge, this is the first result on the application of the HAM with GA to solve models of the time-dependent Emden-Fowler type of equations and wave-type equation with singular behaviors. The HAM with GA has been successfully applied to solve models of the time-dependent Emden-Fowler type of equations and wave-type equation with singular behaviors. The HAM with GA has worked effectively to handle these models giving it a wider applicability. The proposed scheme of HAM has been applied directly without any need for transformation formulae or restrictive assumptions. The solution process of HAM is compatible with those methods in the literature providing analytical approximation such as ADM.

The approach of HAM with GA has been tested by employing the method to obtain approximate-exact solutions of six examples. The results obtained in all cases demonstrate the reliability and the efficiency of this method.

\section{Acknowledgements}

We would like to thank the referees for their careful review of our manuscript.

\section{References}

[1] Davis, H.T. (1962) Introduction to Nonlinear Differential and Integral Equations. Dover Publications, New York.

[2] Chandrasekhar, S. (1967) Introduction to the Study of Stellar Structure. Dover Publications, New York.

[3] Richardson, O.U. (1921) The Emission of Electricity from Hot Bodies. London.

[4] Adomian, G., Rach, R. and Shawagfeh, N.T. (1995) On the Analytic Solution of Lane-Emden Equation, Found. Physics Letters, 8, 161-181. 
https://doi.org/10.1007/BF02187585

[5] Shawagfeh, N.T. (1993) Nonperturbative Approximate Solution for Lane-Emden Equation. Journal of Mathematical Physics, 34, 4364-4369.

https://doi.org/10.1063/1.530005

[6] Wazwaz, A.M. (2001) A New Method for Solving Differential Equations of the Lane-Emden Type. Applied Mathematics and Computation, 118, 287-310.

[7] Wazwaz, A.M. (2005) Adomian Decomposition Method for a Reliable Treatment of the Emden-Fowler Equation. Applied Mathematics and Computation, 161, 543-560.

[8] Wazwaz, A.M. (2005) Analytical Solution for the Time-Dependent Emden-Fowler Type of Equations by Adomian Decomposition Method. Applied Mathematics and Computation, 166, 638-651.

[9] Yıldırım, A. and Öziş, T. (2009) Solutions of Singular IVPs of Lane-Emden Type by the Variational Iteration Method. Nonlinear Analysis, 70, 2480-2484.

[10] Dehghan, M. and Shakeri, F. (2008) Approximate Solution of a Differential Equation Arising in Astrophysics Using the Variational Iteration Method. New Astronomy, 13, 53-59.

[11] Liao, S.J. (2003) Beyond Perturbation: Introduction to the Homotopy Analysis Method. Chapman and Hall, CRC Press, Boca Raton. https://doi.org/10.1201/9780203491164

[12] He, J.H. (2003) Homotopy Perturbation Method: A New Nonlinear Analytical Technique. Applied Mathematics and Computation, 135, 73-79.

[13] Gen, M. and Cheng, R. (1997) Genetic Algorithms and Engineering Design. John Wiley \& Sons, Hoboken.

[14] Melanie, M. (1998) An Introduction to Genetic Algorithms. MIT Press, Cambridge.

[15] Adomian, G. (1989) Nonlinear Stochastic Systems Theory and Applications to Physics. Kluwer Academic Publishers, Dordrecht. https://doi.org/10.1007/978-94-009-2569-4

[16] Wazwaz, A.M. (2000) A New Algorithm for Calculating Adomian Polynomials for Nonlinear Operators. Applied Mathematics and Computation, 111, 53-69.

Submit or recommend next manuscript to SCIRP and we will provide best service for you:

Accepting pre-submission inquiries through Email, Facebook, LinkedIn, Twitter, etc. A wide selection of journals (inclusive of 9 subjects, more than 200 journals)

Providing 24-hour high-quality service

User-friendly online submission system

Fair and swift peer-review system

Efficient typesetting and proofreading procedure

Display of the result of downloads and visits, as well as the number of cited articles

Maximum dissemination of your research work

Submit your manuscript at: http://papersubmission.scirp.org/

Or contact am@scirp.org 